\title{
Nonlocal spin Hall effect and spin-orbit interaction in nonmagnetic metals
}

\author{
S. Takahashi* , S. Maekawa \\ Institute for Materials Research, Tohoku University, Sendai, 980-8577, Japan \\ CREST, Japan Science and Technology Agency, Kawaguchi, 332-0012, Japan \\ Received 12 June 2005; revised 13 June 2005; accepted 14 June 2005
}

\begin{abstract}
Spin Hall effect in a nonlocal spin-injection device is theoretically studied. Using a nonlocal spin-injection technique, a pure spin current is created in a nonmagnetic metal $(\mathrm{N})$. The spin current flowing in $\mathrm{N}$ is deflected by spin-orbit scattering to induce the Hall current in the transverse direction and accumulate charge at the edges of $\mathrm{N}$, yielding the spin-current induced Hall effect. We propose a method for extracting the spin-orbit coupling parameter in nonmagnetic metals via the nonlocal spin-injection technique. (c) 2021 Elsevier B.V. All rights reserved.
\end{abstract}

PACS: 72.25.-b; 72.25.Ba; 72.25.Mk; 85.75.Nn

Keywords: spin Hall effect; spin injection; spin current; nonlocal spin transport

There has been growing interest in spin transport in magnetic nanostructures, because of potential applications to spin electronic devices [1]. Recent experimental studies have demonstrated that the spin polarized carriers injected from a ferromagnet $(\mathrm{F})$ into a nonmagnetic material $(\mathrm{N})$ such as a normal metal $[2,3,4,5]$ and superconductor $[6,7]$ create a spin accumulation in N. In this paper, we consider a nonlocal spin-injection Hall device, and discuss the anomalous Hall effect (AHE) in the presence of spin current (or charge current) flowing in $\mathrm{N}$, taking into account side jump and skew scattering.

The basic mechanism for AHE is the spin-orbit interaction in N, which causes a spin-asymmetry in the scattering of conduction electrons by impurities; up-spin electrons are preferentially scattered in one direction and down-spin electrons in the opposite direction. Spin injection techniques makes it possible to induce AHE in nonmagnetic conductors. When spin-polarized electrons are injected from $\mathrm{F}$ to $\mathrm{N}$, these electrons moving in $\mathrm{N}$ are deflected by the spinorbit scattering to induce the Hall current in the transverse direction and accumulate charge at the edges of $\mathrm{N}$, yielding the spin-current induced spin Hall effect (SHE) $[8,9,10]$.

Using the Boltzmann transport equations which incorporates the spin-asymmetric scattering of conduction electrons by nonmagnetic impurities in $\mathrm{N}$ within the Born approximation, we can derive the "total" spin and charge currents flowing in $\mathrm{N}[10,11]$

\footnotetext{
* Corresponding author. S. Takahashi

Email address: takahasi@imr.tohoku.ac.jp (S. Takahashi).
}

$\mathbf{J}_{s}=\mathbf{j}_{s}+\mathbf{j}_{s}^{\mathrm{H}}, \quad \mathbf{J}_{q}=\mathbf{j}_{q}+\mathbf{j}_{q}^{\mathrm{H}}$.

where $\mathbf{j}_{s}=-\left(\sigma_{\mathrm{N}} / e\right) \nabla \delta \mu_{\mathrm{N}}$ and $\mathbf{j}_{q}=\sigma_{\mathrm{N}} \mathbf{E}$ are the longitudi$n a l$ spin and Ohmic currents, $\sigma_{\mathrm{N}}=2 e^{2} N(0) D$ is the electrical conductivity, $\delta \mu_{\mathrm{N}}=\frac{1}{2}\left(\mu_{\mathrm{N}}^{\uparrow}-\mu_{\mathrm{N}}^{\downarrow}\right)$ is the chemical potential shift, $\mu_{\mathrm{N}}^{\sigma}$ is the chemical potential of electrons with spin $\sigma$, and $D$ is the diffusion constant. The second terms in Eq. (1) are the transverse spin and charge Hall currents caused by spin-orbit scattering:

$\begin{aligned} \mathbf{j}_{s}^{\mathrm{H}}=\alpha_{\mathrm{H}}\left[\hat{\mathbf{z}} \times \mathbf{j}_{q}\right] & =\alpha_{\mathrm{H}} \sigma_{\mathrm{N}}(\hat{\mathbf{z}} \times \mathbf{E}), \\ \mathbf{j}_{q}^{\mathrm{H}}=\alpha_{\mathrm{H}}\left[\hat{\mathbf{z}} \times \mathbf{j}_{s}\right] & =-\frac{\alpha_{\mathrm{H}} \sigma_{\mathrm{N}}}{e}\left(\hat{\mathbf{z}} \times \nabla \delta \mu_{\mathrm{N}}\right),\end{aligned}$

with $\alpha_{\mathrm{H}}=\alpha_{\mathrm{H}}^{\mathrm{SJ}}+\alpha_{\mathrm{H}}^{\mathrm{SS}}$, where $\alpha_{\mathrm{H}}^{\mathrm{SJ}}=\hbar \bar{\eta}_{\mathrm{so}} /(3 m D)$ is the side jump (SJ) contribution, and $\alpha_{\mathrm{H}}^{\mathrm{SS}}=(2 \pi / 3) \bar{\eta}_{\mathrm{so}} N(0) V_{\mathrm{imp}}$ is the skew scattering (SS) contribution, $\bar{\eta}_{\mathrm{so}}=k_{\mathrm{F}}^{2} \eta_{\mathrm{so}}$ is the dimensionless spin-orbit coupling parameter, $k_{\mathrm{F}}$ is the Fermi momentum, and $V_{\text {imp }}$ is the impurity potential.

Equations (2) and (3) indicate that the spin current $\mathbf{j}_{s}$ induces the transverse charge current (charge Hall current) $\mathbf{j}_{q}^{\mathrm{H}}$, whereas the charge current $\mathbf{j}_{q}$ induces the transverse spin current (spin Hall current) $\mathbf{j}_{s}^{\mathrm{H}}$. Equation (1) is expressed in the matrix forms

$$
\begin{aligned}
& {\left[\begin{array}{l}
J_{q, x} \\
J_{s, y}
\end{array}\right]=\left[\begin{array}{cc}
\sigma_{x x} & -\sigma_{x y} \\
\sigma_{x y} & \sigma_{x x}
\end{array}\right]\left[\begin{array}{c}
E_{x} \\
-\nabla_{y} \delta \mu_{\mathrm{N}} / e
\end{array}\right],} \\
& {\left[\begin{array}{l}
J_{s, x} \\
J_{q, y}
\end{array}\right]=\left[\begin{array}{cc}
\sigma_{x x} & -\sigma_{x y} \\
\sigma_{x y} & \sigma_{x x}
\end{array}\right]\left[\begin{array}{c}
-\nabla_{x} \delta \mu_{\mathrm{N}} / e \\
E_{y}
\end{array}\right],}
\end{aligned}
$$


where $\sigma_{x x}=\sigma_{\mathrm{N}}$ is the longitudinal conductivity and $\sigma_{x y}$ is the Hall conductivity contributed from SJ and SS: $\sigma_{x y}=$ $\left(\alpha_{\mathrm{H}}^{\mathrm{SJ}}+\alpha_{\mathrm{H}}^{\mathrm{SS}}\right) \sigma_{\mathrm{N}}=\sigma_{x y}^{\mathrm{SJ}}+\sigma_{x y}^{\mathrm{SS}}$ with

$\sigma_{x y}^{\mathrm{SJ}}=\frac{e^{2}}{\hbar} \eta_{\mathrm{so}} n_{e}, \quad \sigma_{x y}^{\mathrm{SS}}=\alpha_{\mathrm{H}}^{\mathrm{SJ}} \frac{n_{e}}{n_{\mathrm{imp}}}\left[N(0) V_{\mathrm{imp}}\right]^{-1}$,

where $n_{e}$ is the carrier (electron) density and $n_{\mathrm{imp}}$ is the impurity concentration. Note that $\sigma_{x y}^{\mathrm{SJ}}$ is independent of impurity concentration $n_{\text {imp }}$.

The ratio of the SJ and SS Hall contributions is

$\frac{\sigma_{x y}^{\mathrm{SJ}}}{\sigma_{x y}^{\mathrm{SS}}}=2 \frac{n_{\mathrm{imp}}}{n_{e}} N(0) V_{\mathrm{imp}}=\frac{3}{4 \pi} \frac{\hbar}{\epsilon_{\mathrm{F}} \tau_{\mathrm{imp}}} \frac{1}{N(0) V_{\mathrm{imp}}}$,

where $\tau_{\text {imp }}$ is the momentum scattering time and $\epsilon_{\mathrm{F}}$ is the Fermi energy. In ordinary non-magnetic metals, the ratio is very small because $n_{\mathrm{imp}} \ll n_{e}$ and $N(0) V_{\mathrm{imp}} \sim 1$, so that SS gives the dominant contribution to SHE. However, in very dirty metals or in low-carrier materials such as doped semiconductors with $n_{\mathrm{imp}} \sim n_{e}$, the SJ conductivity is comparable to or even larger than the SS conductivity in SHE.

In the following, we consider a spin-injection Hall device shown in Fig. 1, and concentrate on the spin-current induced SHE. The magnetization of $\mathrm{F}$ electrode points to the $z$ direction. When the current $I$ is sent from $\mathrm{F}$ to the left side of $\mathrm{N}$, the spin-polarized electrons are injected to create a pure spin current $\mathbf{j}_{s}$ in $\mathrm{N}$ on the right side, where the total charge current is expressed as

$\mathbf{J}_{q}=-\left(\alpha_{\mathrm{H}} \sigma_{\mathrm{N}} / e\right)\left(\hat{\mathbf{z}} \times \nabla \delta \mu_{\mathrm{N}}\right)+\sigma_{\mathrm{N}} \mathbf{E}$.

where the first term is the Hall current induced by $\mathbf{j}_{s}$, the second term is the Ohmic current induced by surface charge, and $\alpha_{\mathrm{H}} \sim \bar{\eta}_{\mathrm{so}} N(0) V_{\mathrm{imp}}$ (skew scattering). In the open circuit condition in the transverse direction, where $J_{q}^{y}$ vanishes, the nonlocal Hall resistance $R_{\mathrm{H}}=V_{\mathrm{H}} / I$ becomes

$R_{\mathrm{H}}=\frac{1}{2} \alpha_{\mathrm{H}} P_{\mathrm{T}}\left(\rho_{\mathrm{N}} / d_{\mathrm{N}}\right) e^{-L / l_{\mathrm{N}}}$,

in the case of tunnel junction, where $P_{\mathrm{T}}$ is the tunneling spin polarization, $\rho_{\mathrm{N}}$ is the resistivity, $l_{\mathrm{N}}$ is the spindiffusion length, and $d_{\mathrm{N}}$ is the thickness of N. In the case of metallic-contact junction

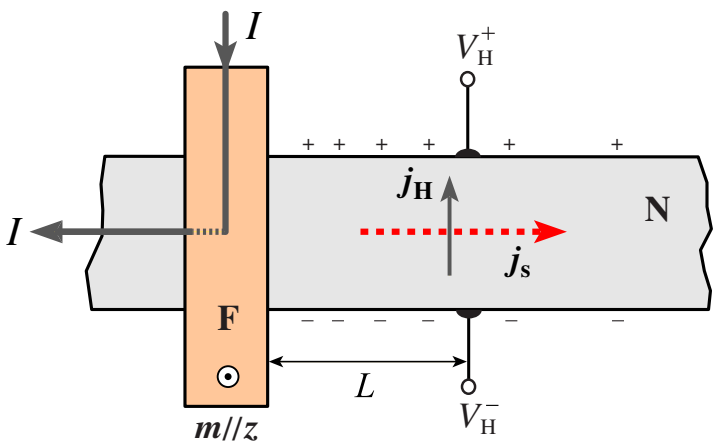

Fig. 1. Spin injection Hall device (top view). The magnetic moment of $\mathrm{F}$ is aligned perpendicular to the plane. The spin-current induced Hall voltage $V_{\mathrm{H}}=V_{\mathrm{H}}^{+}-V_{\mathrm{H}}^{-}$is induced in the transverse direction by injection of pure spin current $\mathbf{j}_{s}$.
Table 1

Spin-orbit coupling parameter $\bar{\eta}_{\mathrm{so}}$ of $\mathrm{Cu}, \mathrm{Al}$, and $\mathrm{Ag}$.

\begin{tabular}{ccccc}
\hline & $l_{\mathrm{N}}(\mathrm{nm})$ & $\rho_{\mathrm{N}}(\mu \Omega \mathrm{cm})$ & $\tau_{\text {imp }} / \tau_{\text {sf }}$ & $\bar{\eta}_{\text {so }}$ \\
\hline $\mathrm{Cu}^{\mathrm{a}}$ & 1000 & 1.43 & $0.70 \times 10^{-3}$ & 0.040 \\
$\mathrm{Cu}^{\mathrm{b}}$ & 1500 & 1.00 & $0.64 \times 10^{-3}$ & 0.037 \\
$\mathrm{Cu}^{\mathrm{c}}$ & 546 & 3.44 & $0.41 \times 10^{-3}$ & 0.030 \\
$\mathrm{Al}^{\mathrm{d}}$ & 650 & 5.90 & $0.36 \times 10^{-4}$ & 0.009 \\
$\mathrm{Ag}^{\mathrm{e}}$ & 195 & 3.50 & $0.50 \times 10^{-2}$ & 0.110 \\
\hline \multicolumn{4}{c}{${ }^{a}$ Ref. [2], ${ }^{b}$ Ref. [3], ${ }^{c}$ Ref. [4], ${ }^{d}$ Ref. [2], ${ }^{e}$ Ref. [5]. }
\end{tabular}

$R_{\mathrm{H}}=\frac{1}{2} \alpha_{\mathrm{H}} \frac{p_{\mathrm{F}}}{1-p_{\mathrm{F}}^{2}}\left(\rho_{\mathrm{N}} / d_{\mathrm{N}}\right) \frac{R_{\mathrm{F}}}{R_{\mathrm{N}}} \sinh ^{-1}\left(L / l_{\mathrm{N}}\right)$,

where $p_{\mathrm{F}}$ is the spin polarization of $\mathrm{F}, R_{\mathrm{N}}$ and $R_{\mathrm{F}}$ are the spin resistances of the $\mathrm{N}$ and $\mathrm{F}$ electrodes: $R_{\mathrm{N}}=\left(\rho_{\mathrm{N}} l_{\mathrm{N}}\right) / A_{\mathrm{N}}$ and $R_{\mathrm{F}}=\left(\rho_{\mathrm{F}} l_{\mathrm{F}}\right) / A_{\mathrm{J}}$ with $A_{\mathrm{N}}$ the cross-sectional area of $\mathrm{N}$ and $A_{\mathrm{J}}$ the contact area between $\mathrm{N}$ and F. Usually, $R_{\mathrm{N}}$ is one or two orders of magnitude larger than $R_{\mathrm{F}}$ [12]. Recently, the spin-current induced AHE have been measured using spin injection techniques $[13,14]$.

It is worthwhile to make the product $\rho_{\mathrm{N}} l_{\mathrm{N}}$, which is related to the spin-orbit coupling parameter $\bar{\eta}_{\mathrm{so}}$ as

$\rho_{\mathrm{N}} l_{\mathrm{N}}=\frac{\sqrt{3} \pi}{2} \frac{R_{\mathrm{K}}}{k_{\mathrm{F}}^{2}} \sqrt{\frac{\tau_{\mathrm{sf}}}{\tau_{\mathrm{imp}}}}=\frac{3 \sqrt{3} \pi}{4} \frac{R_{\mathrm{K}}}{k_{\mathrm{F}}^{2}} \frac{1}{\bar{\eta}_{\mathrm{so}}}$,

where $R_{\mathrm{K}}=h / e^{2} \sim 25.8 \mathrm{k} \Omega$ and $\tau_{\text {sf }}$ is the spin-flip scattering time. The formula (11) provides a method for extracting the physical parameters of spin-orbit scattering in nonmagnetic metals. Using experimental data of $\rho_{\mathrm{N}}$ and $l_{\mathrm{N}}$ in Eq. (11), we obtain the value of the spin-orbit coupling parameter $\bar{\eta}_{\mathrm{so}}=0.01-0.04$ in $\mathrm{Cu}, \mathrm{Al}$, and $\mathrm{Ag}$ as listed in Table 1. Therefore, Eqs. (9) and (10) yields $R_{\mathrm{H}}$ of the order of $0.1-1 \mathrm{~m} \Omega$, indicating that the spin-current induced SHE is observable by using nonlocal spin-injection Hall devices.

This work was supported by the NAREGI Nanoscience Project, Japan.

\section{References}

[1] Concept in Spin Electronics, edited by S. Maekawa (Oxford Univ Press, 2006).

[2] F. J. Jedema et al., Nature (London) 410 (2001) 345; 416 (2002) 713; Phys. Rev. B 67 (2003) 085319.

[3] T. Kimura et al., Appl. Phys. Lett. 85, 3795 (2004); Phys. Rev. B 72 (2005) 014461; Phys. Rev. Lett. 96 (2006) 037201.

[4] S. Garzon et al., Phys. Rev. Lett. 94 (2005) 176601.

[5] R. Godfrey and M. Johnson, Phys. Rev. Lett. 96 (2006) 136601.

[6] M. Urech et al., J. Appl. Phys. 99, 08M513 (2006).

[7] K. Miura et al., Jpn. J. Appl. Phys. 45, 2888 (2006).

[8] J. E. Hirsch, Phys. Rev. Lett. 83 (1999) 1834.

[9] S. Zhang, Phys. Rev. Lett. 85 (2001) 393.

[10] S. Takahashi and S. Maekawa, Phys. Rev. Lett. 88 (2002) 116601.

[11] S. Takahashi et al., Chapter 8 in Concept in Spin Electronics, edited by S. Maekawa (Oxford Univ Press, 2006).

[12] S. Takahashi and S. Maekawa, Phys. Rev. B 67 (2003) 052409.

[13] T. Kimura et al., J. Magn. Magn. Mater. 272-276 (2004) e1333.

[14] H. Saitoh et al., Appl. Phys. Lett. 88 (2006) 182509. 